\title{
Fruit Detachment in Rabbiteye Blueberry: Abscission and Physical Separation
}

\author{
Tripti Vashisth and Anish Malladi ${ }^{1,2}$ \\ Department of Horticulture, 1111 Miller Plant Sciences, University of Georgia, Athens, GA 30602
}

\begin{abstract}
AdDITIONAL INDEX wORDS. abscission agent, abscission zone, mechanical harvesting, plant growth regulators, Vaccinium sp.
Abstract. A better understanding of fruit detachment and the processes mediating it is essential to improve the efficiency of mechanical harvesting in blueberry (Vaccinium sp.). In blueberry, fruit detachment may occur either at the point of attachment of the pedicel to the peduncle [peduncle-pedicel junction (PPJ)] or at the point of attachment of the pedicel to the fruit [fruit-pedicel junction (FPJ)]. The fruit detachment responses of the PPJ and the FPJ to different conditions are not entirely clear. Additionally, whether fruit detachment at these junctions is mediated by the physiological process of abscission or through physical separation of the organ from the parent plant is not well understood. In this study, a series of experiments were performed to determine the abscission zone (AZ) corresponding to the point of mature fruit detachment and to determine whether fruit detachment occurs as a result of abscission or physical separation in rabbiteye blueberry (Vaccinium ashei). Anatomical studies indicated the presence of an AZ at the PPJ. Greater than 92\% of the natural detachment of mature fruit occurred at the PPJ. The morphology of the fracture plane at the PPJ in naturally detached fruit was even and uniform, consistent with fruit detachment through abscission at this location. Abscission agents such as methyl jasmonate (20 mu) and ethephon $\left(1000 \mathrm{mg} \cdot \mathrm{L}^{-1}\right)$ enhanced the extent of fruit detachment at the PPJ, further indicating that mature fruit detachment through abscission occurred primarily at this location. Additionally, the fracture plane at the PPJ during fruit detachment in response to abscission agent applications was flattened and even, further supporting the conclusion that fruit detachment at this location occurred through abscission. In contrast, the majority of the fruit detachment in response to mechanical shaking occurred at the FPJ. Analysis of the morphology of the fracture plane at the FPJ during detachment in response to mechanical shaking indicated that fruit detachment at this location was associated with extensive tearing and mechanical disruption of cells, consistent with physical separation. Together, data from this study indicate that mature fruit detachment resulting from abscission occurs primarily at the PPJ, whereas fruit detachment during mechanical shaking occurs primarily at the FPJ as a result of physical breakage at this weak junction.
\end{abstract}

Blueberry, a fruit crop indigenous to the United States, was valued at over $\$ 780$ million in 2011 [U.S. Department of Agriculture (USDA), 2012]. Despite its emerging importance, various aspects of blueberry physiology including the process of fruit detachment are not well understood. Enhancing our knowledge of the process of fruit detachment can greatly aid in developing methods to increase the efficiency of mechanical harvesting, which can in turn reduce the costs associated with blueberry production (Austin and Williamson, 1977; Howell et al., 1976; Mainland et al., 1975; Takeda et al., 2008).

Fruit detachment may occur either through the physiological process of abscission or through tearing and physical separation of the fruit from the parent plant. Abscission involves the programmed separation of entire organs in response to plant developmental cues and various types of biotic and abiotic stress. Abscission occurs at specific regions within the plant termed abscission zones (Roberts et al., 2000, 2002; Sexton and Roberts, 1982). The cells in the AZ are morphologically and biochemically distinct before the initiation of the process of separation. The AZs generally consist of one to many layers of small, rounded, densely cytoplasmic cells at the union of the organ destined for detachment and the main body of the plant (Goren, 1993; Roberts et al., 2002; Sexton and Roberts, 1982).

Received for publication 17 Dec. 2012. Accepted for publication 28 Jan. 2013. This research was supported by a grant from the U.S. Department of Agriculture, Specialty Crops Research Initiative (SCRI): 2008-51180-19579.

We thank Lisa K. Johnson for help with experiments in this study.

${ }^{1}$ Assistant Professor.

${ }^{2}$ Corresponding author. E-mail: malladi@uga.edu.
The AZ cells generally contain large deposits of starch and have highly branched plasmodesmata (Sexton and Roberts, 1982). These cells also display several ultrastructural changes during differentiation (Goren, 1993; Iwahori and Van Steveninck, 1976; Patterson, 2001; Roberts et al., 2002; Stösser et al., 1969; Webster, 1968). The progression of abscission at the pre-formed $\mathrm{AZs}$ involves multiple phases such as a gain in competence to respond to abscission signals, activation of the $\mathrm{AZ}$ resulting in the initiation of cell separation in response to the abscission signals, and cell separation followed by formation of a protective layer (Patterson, 2001).

Fruit crops may possess multiple AZs where fruit detachment can occur, often depending on the maturity of the separating organ. For example, in citrus (Citrus sp.) fruit, two AZs are associated with the fruit: 1) the shoot-peduncle AZ (AZ-A); and 2) the peduncle-fruit AZ (AZ-C) (Goren, 1993). Although the AZ-A is active during early fruit development, the $\mathrm{AZ}-\mathrm{C}$ is the active zone associated with mature fruit detachment (Goren, 1993; Kazokas and Burns, 1998). Similarly, in the sour cherry (Prunus cerasus), two AZs have been described, one at the junction of the pedicel and the peduncle and another at the union of the pedicel and the fruit (Stösser et al., 1969; Wittenbach and Bukovac, 1974). Mature sour cherry fruit abscission occurs primarily at the AZ located at the junction of the pedicel and the fruit (Stösser et al., 1969; Wittenbach and Bukovac, 1974). Also in sweet cherry (Prunus avium), immature fruit detachment occurs at the pedicel-peduncle AZ, whereas mature fruit detachment occurs at the receptacle-fruit AZ (Wittenbach and Bukovac, 1972). 
Plant organs can also separate from the plant as a result of mechanical breakage and cell disruption within weak points of attachment in response to physical force instead of abscission. During hand or mechanical harvesting, the fruit may be separated from the plant as a result of cell rupture and breakage at the $\mathrm{AZ}$ or at other physically weak points of attachment of the fruit to the plant. For example, mechanical harvesting in sweet cherry can result in mature fruit detachment between the pedicel and the peduncle (Norton et al., 1962), whereas mature fruit normally abscise at the receptacle-fruit AZ (Wittenbach and Bukovac, 1972).

Fruit detachment in blueberry can occur at the point of attachment of the pedicel to the peduncle or at the point of attachment of the pedicel to the berry. Gough and Litke (1980) reported the presence of an $\mathrm{AZ}$ at the point of attachment of the pedicel to the berry and indicated that mature fruit detachment in northern highbush blueberry (Vaccinium corymbosum) occurs primarily at this location. This junction is referred to hereafter as the fruit-pedicel junction (FPJ). Hand harvesting and the majority of mechanical harvesting of blueberry cultivars typically result in fruit separation at the FPJ (Howell et al., 1976; Takeda et al., 2008). Recent studies indicate that fruit drop in response to the application of abscission agents such as ethephon (2-chloroethylphosphonic acid) and methyl jasmonate (MeJa) occurs primarily at the point of attachment of the pedicel to the peduncle in different types of blueberries (Malladi et al., 2012). This junction is referred to hereafter as the pedunclepedicel junction (PPJ). The point of natural detachment of mature fruit is not well understood. Additionally, it is unclear whether fruit separation at these junctions occurs as a result of abscission or as a result of physical tissue disruption.

A better understanding of the point of fruit detachment and the processes mediating it in blueberry is essential to improve the efficiency of mechanical harvesting. Such information can aid in determining the applicability of abscission agents as harvest aids and in the breeding and selection of genotypes better suited for mechanical harvesting. Hence, the main objectives of this study were to determine the point of mature fruit detachment in blueberry and to determine if fruit detachment occurs through the physiological process of abscission or through physical separation. To achieve these objectives, the anatomy of the points of fruit detachment, natural fruit detachment, fruit detachment in response to abscission agents, and fruit detachment in response to mechanical shaking were investigated in rabbiteye blueberry.

\section{Materials and Methods}

ANATOMY OF THE PEDUNCLE-PEDiCEL JUNCTION AND THE FRUIT-PEDICEL JUNCTION. To study the anatomy of the potential points of fruit detachment, the PPJ and the FPJ tissues from mature (blue/black) fruit ready for harvest were collected from six-year-old 'Powderblue' rabbiteye blueberry plants $(\mathrm{n}=4)$ grown at the University of Georgia, Horticulture Farm, Watkinsville, GA (UGHF) in 2011. The fruit were collected along with the fruiting branch and the PPJ and the FPJ tissues were dissected. The tissues were fixed in FAA (50\% ethyl alcohol, 10\% formalin, 5\% acetic acid) and stored at room temperature until further analysis. Two to three PPJ and FPJ samples from each replicate plant were used in this study. The samples were removed from the FAA solution and rinsed thoroughly in deionized water. The samples were dehydrated through a graded ethanol series. Paraffin infiltration and embedding were performed as described in Ruzin (1999) with the modification of using Histoclear (Electron Microscopy Science, Hatfield, PA) during the infiltration process. Longitudinal sections $(10 \mu \mathrm{m})$ were collected using a rotary microtome. After removal of paraffin from the sections using Histoclear, the sections were rehydrated and stained with $1 \%$ aqueous toluidine blue. Images of the sections were captured using a light microscope (BX51; Olympus, Center Valley, PA) fitted with a digital camera (DP70; Olympus).

Natural fruit detachment. Mature (four- to six-year-old) rabbiteye blueberry plants of 'Premier' and 'Briteblue' grown at UGHF were used in this study. This study was performed in 2011 and in 2012 with both of the genotypes $(n=8$ plants for 'Premier' in 2011 and $n=4$ plants in all other experiments). A $1-m^{2}$ region (square) was marked underneath each plant and all fruit debris were removed from this region. The number of mature fruit that naturally detached from the plant and remained within the marked area was counted at regular intervals. From among these fruit, the proportion that detached along with the pedicel (i.e., at the PPJ) was determined. After each observation, the detached fruit were removed from within the marked area. Data collection was performed for 2 to 3 weeks in each growing season. Cumulative fruit detachment at the PPJ over this period is presented here.

In 2012, the morphology of the fracture plane in naturally detached fruit was studied in 'Briteblue'. The area under the bushes was cleared and the bushes were manually monitored over a period of several hours until at least two mature fruit per replicate plant $(n=4)$ naturally detached from the plant. These fruit were collected immediately after separation from the plant. All of the detached fruit separated at the PPJ. The pedicel from the detached fruit was immediately separated from the berry, fixed in 5\% glutaraldehyde:0.1 M potassium phosphate buffer (1:1), and stored at $4{ }^{\circ} \mathrm{C}$ until further analysis using scanning electron microscopy (SEM). The pedicel end of the PPJ (pedicel part detached from the peduncle) was observed in this analysis. Samples were processed for SEM according to Mims (1981). Briefly, the samples were cut into $\approx 1$ - to $2-\mathrm{mm}$ upright sections and were rinsed three times with the fixative buffer, 5\% glutaraldehyde:0.1 M potassium phosphate buffer (1:1), for $15 \mathrm{~min}$. The samples were immediately treated with osmium tetroxide for $2 \mathrm{~h}$ and rinsed. The rinsed samples were dehydrated through a graded ethanol series. For critical point drying, the ethanol was replaced by liquid $\mathrm{CO}_{2}$, which was brought to the critical point in the Autosamdri-814 Critical Point Dryer (Tousimis Research Corp., Rockville, MD). Subsequently, samples mounted on aluminum stubs were coated with gold in the SPI-Module Sputter Coater and Carbon-Coater (SPI Supplies/Structure Probe, West Chester, PA). Samples were observed using a scanning electron microscope (1450EP; Carl Zeiss MicroImaging, Thornwood, NY).

Fruit DETACHMENT IN RESPONSE TO ABSCISSION AGENT APPLICATIONS AND MECHANICAL SHAKING. Mature (five-yearold) rabbiteye blueberry plants of 'Briteblue' grown at UGHF were used in this study in 2011. The experiment was designed as a completely randomized design with three treatments and three replicates (individual plants). The treatments were: 1) control; 2) MeJa (20 mm; Sigma-Aldrich, St. Louis, MO); and 3) ethephon (1000 $\mathrm{mg} \cdot \mathrm{L}^{-1}$; Bayer CropScience, Kansas City, MO). These abscission agent treatments have been previously shown to induce rapid fruit detachment in multiple 
rabbiteye blueberry genotypes (Malladi et al., 2012). The treatments were performed when the majority $(\approx 75 \%)$ of the fruit on the plant was mature. All treatments, including the control, were applied along with $0.15 \%$ of the adjuvant (Latron B-1956; Rohm and Haas, Philadelphia, PA) and were performed using a hand pump sprayer until runoff $\approx 0830 \mathrm{HR}$. The average daily temperature on the day of application was $27^{\circ} \mathrm{C}$. At $24 \mathrm{~h}$ after treatment, two branches per plant with $\approx 50$ fruit each were shaken using a handheld mechanical shaker (described in Malladi et al., in press) until all of the berries were detached. Although MeJa- and ethephon-treated plants required 3 and $3.5 \mathrm{~s}$ of mechanical shaking, respectively, $14 \mathrm{~s}$ of shaking was required for fruit detachment from the control plants (Malladi et al., in press). The immature fruit were removed before mechanical shaking. The detached berries were captured in a catch frame and the proportion of fruit that detached along with the pedicel at the PPJ was determined. An additional branch on each plant was tagged and the fruit number was counted to determine the extent of drop as a result of the treatments. After $72 \mathrm{~h}$ of treatment, 13\% fruit drop was observed in the control, whereas MeJa and ethephon treatments resulted in $65 \%$ and $66 \%$ fruit drop, respectively.

In 2012, mature seven-year-old rabbiteye blueberry plants of 'Powderblue' grown at UGHF were used to study the morphology of the fracture plane during fruit detachment in response to abscission agent applications $(n=4)$. This experiment was designed as a completely randomized design with three treatments: 1) control; 2) MeJa (20 mM); and 3) ethephon $\left(1000 \mathrm{mg} \cdot \mathrm{L}^{-1}\right)$. All treatments included $0.15 \%$ of the adjuvant (Latron B-1956) and were performed using a hand pump sprayer until runoff around $0900 \mathrm{HR}$. The average daily temperature on the day of application was $28^{\circ} \mathrm{C}$. At $24 \mathrm{~h}$ after treatment, the pedicels were separated manually from the peduncle or from the mature fruit to obtain the PPJ and the FPJ fracture planes, respectively. Three samples of the PPJ and the FPJ were collected from each replicate plant. These samples were immediately fixed in 5\% glutaraldehyde:0.1 M potassium phosphate buffer $(1: 1)$, stored at $4{ }^{\circ} \mathrm{C}$, and used for SEM analysis, as described previously. The pedicel ends of the PPJ and the FPJ (pedicel part detached from the fruit) were observed in this study.

Fruit Detachment in RESPONSE TO MECHANICAL SHAKING. Mature (six-year-old) rabbiteye blueberry plants of 'Briteblue' grown at UGHF were used to understand fruit detachment in response to mechanical shaking in 2012. One branch per plant with $\approx 50$ fruit was shaken using a handheld mechanical shaker (Malladi et al., in press) for $5 \mathrm{~s}$ and the detached fruit were captured in a catch frame $(n=3)$. The proportion of the detached fruit that separated at the PPJ was determined. Additionally, the PPJ tissues were collected from the mature berries that separated with the pedicel attached (collected in a catch frame), whereas the FPJ tissues were collected from the pedicels that were left on the branch after the detachment of the fruit. Two PPJ and FPJ tissues were dissected and collected from each replicate. These samples were immediately fixed in 5\% glutaraldehyde:0.1 M potassium phosphate buffer $(1: 1)$ and were stored at $4{ }^{\circ} \mathrm{C}$ until further analysis. The pedicel ends of these junctions were used for SEM analysis, as described previously, to better understand the mechanism of fruit detachment resulting from mechanical shaking.

STAtistical analysis. The data from the study on fruit detachment in response to abscission agent applications and mechanical shaking were analyzed using analysis of variance followed by mean separation using Fisher's least significant difference $(\alpha=0.05)$. All analyses were performed using SigmaPlot 11 (Systat Software, San Jose, CA). Data collected from two branches per plant were treated as subsamples and averaged within the replicate.

\section{Results and Discussion}

Anatomy of The PPJ AND The FPJ. At the point of attachment of the pedicel to the peduncle (PPJ), multiple layers of typically small and isodiametric cells were observed on either side of the vascular tissue (Fig. 1A). These cells were distinct from the surrounding cells within the peduncle and pedicel, which were generally larger in size. Indentations were observed on either side of the point of attachment and the cells within this region were also characteristically smaller, especially within the peduncle. AZs are typically characterized by the presence of such multiple layers of small rounded cells, which also display intense staining (Roberts et al., 2002; Sexton and Roberts, 1982). Hence, these observations are consistent with the presence of an AZ at the PPJ and suggest that progression of fruit detachment at this location can occur through the physiological process of abscission. However, a line of fracture or cell rupture was not observed within the PPJ samples analyzed here, indicating that the progression of cell separation had not yet been initiated at this region in these samples. Additionally, a zone of cell separation was not apparent across the vascular bundles connecting the pedicel and the peduncle, suggesting that detachment along this region may occur through mechanical fracture.

A clear AZ was not apparent at the FPJ (Fig. 1B). The majority of the cells at this junction appeared to be large and rounded and similar to the neighboring cells. Although an indentation between the pedicel and the fruit was observed toward the periphery, no clear plane of separation was evident in this region. These observations are not consistent with the

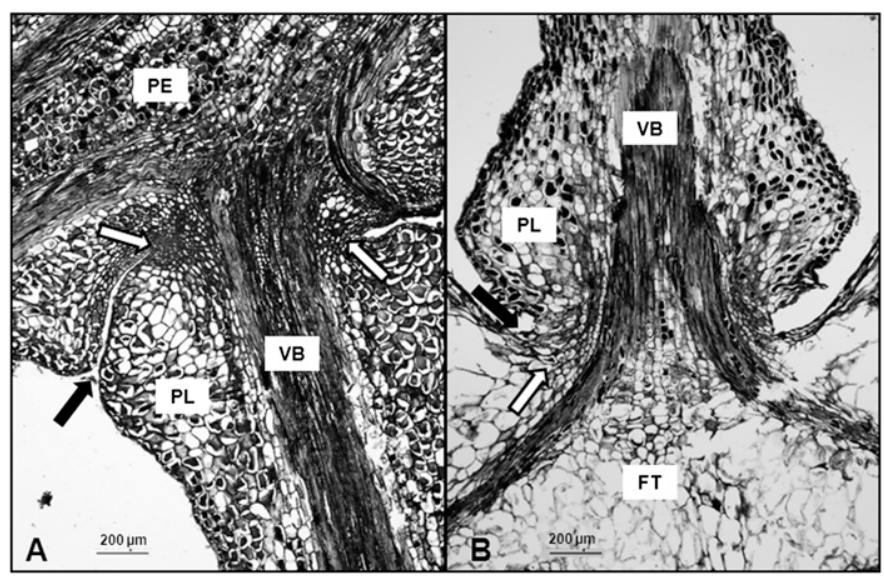

Fig. 1. Anatomy of the peduncle-pedicel and the fruit-pedicel junctions in 'Powderblue' rabbiteye blueberry. (A) The peduncle-pedicel junction is shown. The black arrow indicates the indentation at the point of attachment of the peduncle (PE) and the pedicel (PL). The white arrows on either side of the vascular bundle (VB) indicate multiple layers of small rounded cells within the abscission zone. (B) The fruit-pedicel junction is presented. The black arrow indicates the indentation at the point of attachment of the pedicel (PL) and the fruit (FT). The white arrow indicates normal size cells next to the vascular bundle $(\mathrm{VB})$ at the junction of the pedicel and the fruit $(\mathrm{bar}=200 \mu \mathrm{m})$. 
presence of an AZ at the union of the pedicel and the fruit. However, Gough and Litke (1980) reported the presence of an AZ at the FPJ in northern highbush blueberry. It is possible that these differences are the result of differences between the Vaccinium species used in these studies. Anatomical analysis of the FPJ across different species may be required to address the possibility of the presence of an AZ at this location in other Vaccinium species. Such a study may be complemented by the analysis of the localized expression of genes and immunohistochemical analysis using markers specifically associated with the AZs as has been recently described in tomato (Iwai et al., 2012).

NAtURAL FRUit Detachment. The majority of natural fruit detachment occurred at the point of attachment of the pedicel to the peduncle (i.e., at the PPJ) in both of the rabbiteye blueberry cultivars (Fig. 2). In 'Premier', 92\% and 98\% of the berries detached at the PPJ in 2011 and 2012, respectively. In 'Briteblue', 98\% of the fruit detached at the PPJ in both years of this study. A similar trend of natural fruit detachment was also observed in 'Powderblue' (data not shown). These data indicate that natural detachment of mature fruit occurs at the PPJ across the different rabbiteye blueberry genotypes analyzed.

The PPJ was used for SEM analysis of the morphology of the fracture plane because natural fruit detachment occurred primarily at this location. The pedicel end of the PPJ in naturally detached fruit displayed an even fracture plane (Fig. 3). Small and rounded cells were visible at the surface and at the periphery of the fracture plane. These observations are consistent with the involvement of abscission during natural fruit detachment. Cell rupture and breakage and some tearing of the tissues were observed at the center of fracture plane, primarily within the vascular tissue, suggesting that abscission progressed through the disruption of cells within this region. Similarly, breakage of cells within the vascular tissue was associated with the final stages of abscission progression in cherry (Stösser et al., 1969; Wittenbach and Bukovac, 1972). Also, Gough and Litke (1980) reported that final fruit separation in blueberry occurs by rupture and mechanical tearing of the vascular bundles, although these authors indicated that

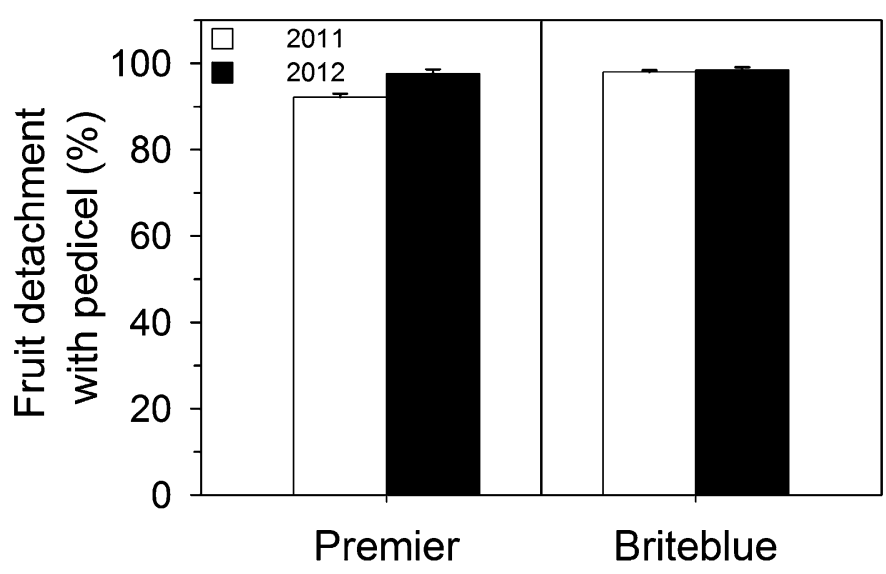

Fig. 2. Cumulative natural detachment of mature fruit at the peduncle-pedicel junction in the rabbiteye blueberry cultivars, Premier and Briteblue in 2011 and 2012. Natural fruit drop within a $1-\mathrm{m}^{2}$ area below the plant was determined at regular intervals for 2 to 3 weeks. The proportion of naturally dropped fruit that detached at the peduncle-pedicel junction is presented. Error bars indicate SE.

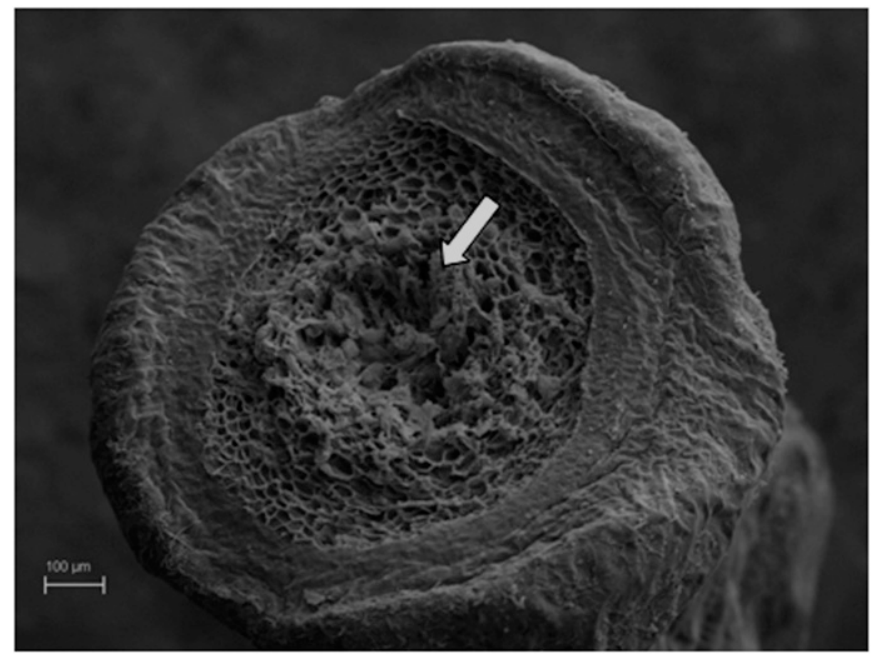

Fig. 3. Morphology of the fracture plane of the pedicel end of the pedunclepedicel junction in naturally detached 'Briteblue' rabbiteye blueberry fruit. Fruit naturally detached from the plant at the peduncle-pedicel junction were collected and the fracture plane at the point of detachment was observed using scanning electron microscopy $(\mathrm{bar}=100 \mu \mathrm{m})$. The arrow indicates the region of extensive cell rupture and breakage within the vascular tissue.

fruit detachment occurred at the FPJ in northern highbush blueberry. Together, the natural fruit detachment data and the SEM analysis support the involvement of the physiological process of abscission during natural fruit detachment in the rabbiteye blueberry cultivars studied.

Fruit detACHMENT in RESPONSE TO ABSCISSION AgENT APPLICATIONS AND MECHANICAL SHAKING. The majority of the fruit in the control treatment detached at the FPJ after mechanical shaking (Fig. 4) in contrast to the point where the majority of natural fruit detachment occurred. Although some

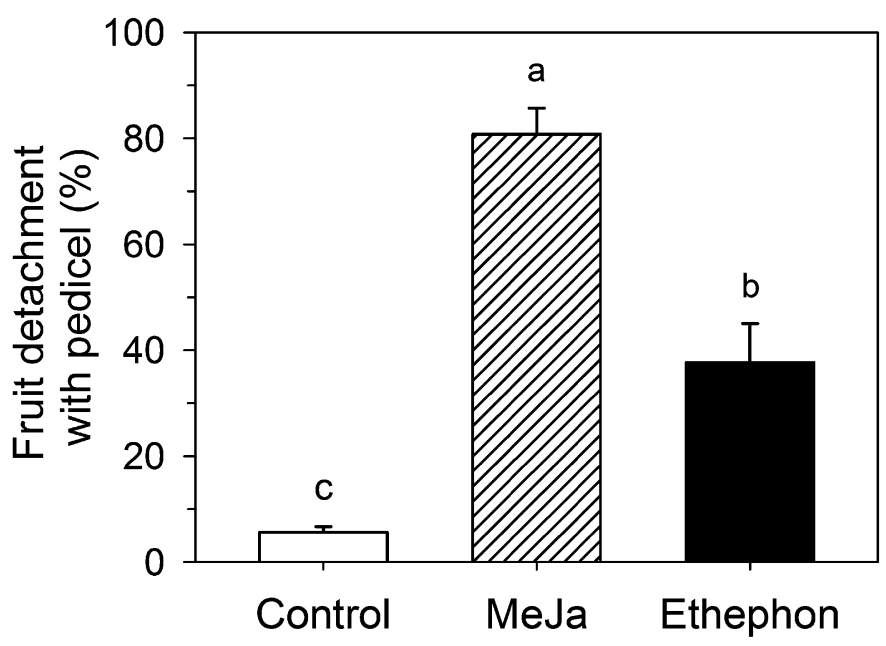

Fig. 4. Effect of abscission agents on the point of fruit detachment in rabbiteye blueberry. Control (adjuvant only), $20 \mathrm{~mm}$ methyl jasmonate (MeJa), and $1000 \mathrm{mg} \cdot \mathrm{L}^{-1}$ ethephon applications were performed on 'Briteblue' blueberry plants $(\mathrm{n}=3)$. At $24 \mathrm{~h}$ after application, individual branches with $\approx 50$ fruit each were shaken using a mechanical shaker. The proportion of detached fruit that retained the pedicel was determined. Analysis of variance was performed followed by mean separation using Fisher's least significant difference $(\alpha=$ 0.05 ). Error bars represent SE. Similar letters above the bars indicate no significant difference between treatments. 
of this difference may be the result of the different modes of fruit detachment, it may also be likely that fruit that naturally detach at the PPJ may be primed for detachment owing to a slightly advanced degree of fruit maturity. Mechanical shaking at $24 \mathrm{~h}$ after the application of the abscission agents, MeJa and ethephon, clearly enhanced the extent of fruit detachment at the PPJ (Fig. 4). The applications of MeJa and ethephon resulted in $81 \%$ and $38 \%$ fruit detachment at the PPJ, respectively, in response to mechanical shaking. Similarly, Malladi et al. (2012) reported that the majority of the fruit detachment in response to MeJa and ethephon treatments occurred at the PPJ in multiple rabbiteye blueberry genotypes as well as in southern highbush blueberry (hybrids largely derived from $V$. corymbosum and $V$. darrowi). Together, these data strongly indicate that fruit detachment in response to abscission agent applications was consistent across different cultivars and species of blueberry and occurred primarily at the PPJ. The difference in the extent of fruit detachment at the PPJ between MeJa and ethephon may be a reflection of the time taken for peak fruit loosening effects of these agents. The applications of MeJa generally resulted in high fruit detachment within $24 \mathrm{~h}$, whereas fruit drop in response to ethephon applications was generally high at $\approx 48 \mathrm{~h}$ after treatment (Malladi et al., 2012). In earlier studies, preharvest treatment of ethephon followed by mechanical harvesting of highbush blueberry was reported to result in less than 5\% "stemmy fruit" (Howell et al., 1976) in contrast to the observations described here and the study of Malladi et al. (2012). In the study by Howell et al. (1976), observations on fruit detachment were performed at $\approx 1$ week after treatment, whereas the observations in the current study were performed at $24 \mathrm{~h}$ after treatment. It may be likely that fruit detachment at later stages (1 week after treatment) does not reflect the direct effects of ethephon on abscission. Alternatively, the differences among these studies may be attributed to the different blueberry genotypes used.

The morphology of the fracture planes on the pedicel ends of the PPJ and the FPJ from the control, MeJa-, and ethephontreated samples was observed after manual separation of the pedicel from the peduncle and the fruit, respectively (Fig. 5). The fracture plane of the pedicel end of the PPJ in the control treatment was uneven and consisted of a large portion of the peduncle tissue (Fig. 5A), indicating that fruit detachment at the PPJ involved extensive mechanical breakage at the junction. The fracture plane of the pedicel end of the PPJ in the ethephon treatment also displayed the presence of parts of the peduncle, although this was lesser than that observed in the control (Fig. 5C). These data suggest that some of the separation in response to ethephon applications was associated with mechanical breakage. In MeJa-treated samples, the pedicel end of the PPJ displayed a smoother fracture plane with a few broken cells toward the center of pedicel (Fig. 5E). The peduncle tissue was not attached to the fracture plane indicating uniform fruit detachment involving lesser mechanical disruption in response to MeJa applications. The morphological features of the fracture plane observed here are consistent with
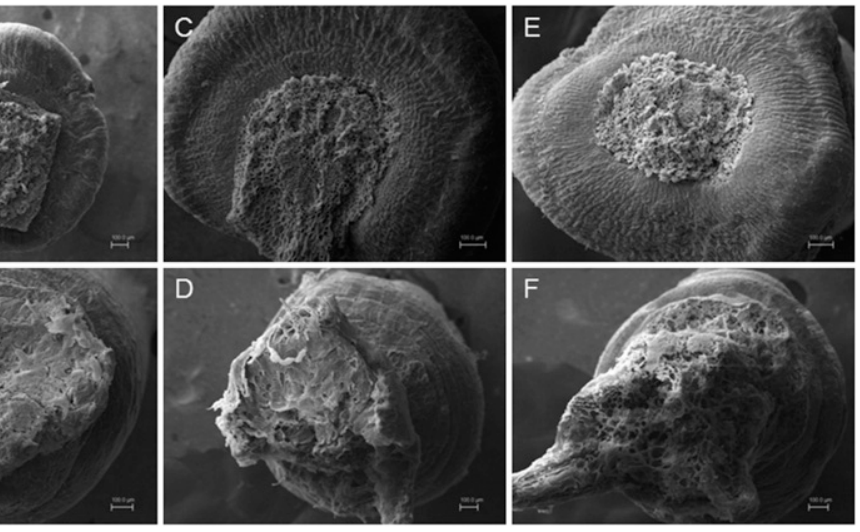

g. 5. Effect of abscission agents on the morphology of the fracture plane of the peduncle-pedice and the fruit-pedicel junctions. Control (A-B), $1000 \mathrm{mg} \cdot \mathrm{L}^{-1}$ ethephon $(\mathbf{C}-\mathbf{D})$, and $20 \mathrm{~mm}$ methyl peduncle-pedicel junction (A, C, and $\mathbf{E})$. Similarly, the pedicel was detached manually from the fruit to obtain the fracture plane of the fruit-pedicel junction $(\mathbf{B}, \mathbf{D}$, and $\mathbf{F})$. The fracture plane of the pedicel end of the junctions was analyzed using scanning electron microscopy $(\mathrm{bar}=100 \mu \mathrm{m})$.

that reported during ethylene-induced citrus leaf abscission (Agustí et al., 2009). In this study, before the ethylene treatment, the fracture plane displayed a ragged surface consisting of broken cell walls, indicating forcible separation at the laminar AZ, similar to that observed in the control treatment in the current study. At $24 \mathrm{~h}$ after ethylene treatment, the $\mathrm{AZ}$ planes were mostly flattened with some breakage within the pith and vascular bundles, similar to that observed in the MeJa treatment in the current study. These observations suggest that fruit detachment at the PPJ in response to the abscission agents, especially MeJa, was associated with the physiological



Fig. 6. Fruit detachment in response to mechanical shaking in 'Briteblue' rabbiteye blueberry. Individual branches were shaken using a mechanical shaker for $5 \mathrm{~s}$. The fruit number before and after shaking was used to determine the extent of total fruit detachment $(n=3)$. Detached fruit were collected in a catch frame and the proportion of fruit that detached at the peduncle-pedicel junction (with the pedicel) was determined. Error bars represent SE. 
process of abscission. In response to the application of the abscission agents, the progression of abscission may be accelerated, resulting in a smoother fracture plane at the PPJ, especially in response to MeJa applications. Differences in the morphology of the fracture plane between the MeJa and ethephon treatments may be attributed to differences in the timing of peak efficacy of the treatments (Malladi et al., 2012).

In the control treatment, the fracture plane of the pedicel end of the FPJ contained parts of the fruit tissue in addition to extensively disrupted cells (Fig. 5B). Neither of the abscission agent treatments altered the appearance of the fracture plane at the pedicel end of the FPJ (Figs. 5D and 5F). These data clearly indicate that the abscission agent applications did not affect detachment characteristics at the FPJ. Hence, fruit detachment at the FPJ in response to mechanical shaking after abscission agent applications was likely associated with mechanical tearing and physical separation of the berry.

Fruit DetaChMENT IN RESPONSE TO MECHANICAL SHAKING. Mechanical shaking for $5 \mathrm{~s}$ resulted in the detachment of $\approx 65 \%$ of the fruit in 'Briteblue' (Fig. 6). Interestingly, the majority of the fruit detachment in response to mechanical shaking occurred at the point of attachment of the pedicel to the fruit (i.e., at the FPJ), whereas only $\approx 14 \%$ of the detached fruit separated at the PPJ. These data indicate that fruit detachment during mechanical shaking occurs at a junction distinct from the point of fruit detachment associated with natural or abscission agentinduced fruit drop.

Among the few fruit that detached at the PPJ during mechanical shaking, the fracture plane of the pedicel end of the PPJ appeared to be flattened and even, similar to that observed during natural fruit detachment (Fig. 7A). The few fruit that detached at the PPJ during mechanical shaking may likely represent mature fruit in which the physiological process of natural abscission had already been initiated. Weakening of this junction resulting from the progression of abscission may aid in the detachment of these fruit at this location on the application of physical force during mechanical shaking. The fracture plane at the pedicel end of the FPJ was also observed using fruit that detached at the FPJ during mechanical shaking. This fracture plane consisted of extensively disrupted cells (Fig. 7B). Importantly, part of the fruit tissue was often found to be attached to the pedicel at this fracture plane. These data indicate that fruit detachment at the FPJ during mechanical shaking occurred as a result of physical breakage and tearing, processes not consistent with abscission.

\section{Conclusions}

Anatomical analyses clearly indicated the presence of an AZ at the PPJ but did not support the presence of an AZ at the FPJ in rabbiteye blueberry. The PPJ appears to be the main point of fruit detachment when the mature fruit naturally drop as a result of the physiological and developmental progression of abscission. This conclusion is supported by the presence of a largely smooth fracture plane along with mild tissue disruption, primarily within the vascular tissue in the naturally detached fruit. The application of abscission agents that may be expected to accelerate the progression of abscission resulted in fruit detachment primarily at the PPJ, further supporting the conclusion that fruit detachment at this junction is associated with abscission. However, mechanical shaking alone resulted in fruit detachment at the FPJ. Such detachment of the fruit was not consistent with abscission but was generally associated with extensive physical disruption of cells. Hence, it is likely that fruit detachment at the FPJ is a result of mechanical breakage and physical separation. The FPJ may present a weak junction and the oscillations of the berry around this point during mechanical shaking may result in physical separation at this junction. Similarly, manual fruit removal may also result in physical separation at this weak junction.

Knowledge of fruit detachment gained from this study has potential implications for mechanical harvesting of blueberry fruit. Abscission agents have been shown to considerably enhance the extent of fruit detachment (Malladi et al., 2012). However, because the physiological process of abscission is involved in such detachment, it is likely that the applications of these agents will result in "stemmy" fruit, which will subsequently require destemming because the presence of the pedicel on the fresh fruit is considered to be a defect that reduces its quality (USDA, 1995). Additionally, breeding programs aiming to improve mechanical harvesting traits may need to select genotypes with stronger pedicel-peduncle junctions so that fruit detachment during mechanical harvesting in such genotypes can occur at the weak union along the FPJ. In conjunction with this, selection for a dry or a small stem scar at this junction may help maintain the post-harvest quality of the fruit.

\section{Literature Cited}

Agustí, J., P. Merelo, M. Cercós, F.R. Tadeo, and M. Talón. 2009. Comparative transcriptional survey between laser-microdissected cells from laminar abscission zone and petiolar cortical tissue during ethylene-promoted abscission in citrus leaves. BMC Plant Biol. 9:127.

Austin, M.E. and R.E. Williamson. 1977. Comparison of harvest methods of rabbiteye blueberries. J. Amer. Soc. Hort. Sci. 102:454-456.

Goren, R. 1993. Anatomical, physiological, and hormonal aspects of abscission in citrus. Hort. Rev. 15:145-182.

Gough, R.E. and W. Litke. 1980. An anatomical and morphological study of abscission in highbush blueberry fruit. J. Amer. Soc. Hort. Sci. 105:335-341.

Howell, G.S., B.G. Stergios, S.S. Stackhouse, H.C. Bittenbender, and C.L. Burton. 1976. Ethephon as a mechanical harvesting aid for highbush blueberries (Vaccinium austral Small). J. Amer. Soc. Hort. Sci. 101:111-115. 
Iwahori, S. and R.F. Van Steveninck. 1976. Ultrastructural observation of lemon fruit abscission. Sci. Hort. 4:235-246.

Iwai, H., A. Terao, and S. Satoh. 2012. Changes in distribution of cell wall polysaccharides in floral and fruit abscission zones during fruit development in tomato (Solanum lycopersicum). J. Plant Res. doi: 10.1007/s10265-012-0536-0.

Kazokas, W.C. and J.K. Burns. 1998. Cellulase activity and gene expression in citrus fruit abscission zones during and after ethylene treatment. J. Amer. Soc. Hort. Sci. 123:781-786.

Mainland, C.M., L.J. Kushman, and W.E. Ballinger. 1975. The effect of mechanical harvesting on yield, quality of fruit and bush damage of highbush blueberry. J. Amer. Soc. Hort. Sci. 100:129-134.

Malladi, A., T. Vashisth, and L.K. Johnson. 2012. Ethephon and methyl jasmonate affect fruit detachment in rabbiteye and southern highbush blueberry. HortScience 47:1745-1749.

Malladi, A., T. Vashisth, and S. NeSmith. 2013. Development and evaluation of a portable, hand-held mechanical shaker to study fruit detachment in blueberry. HortScience 48 (in press).

Mims, C.W. 1981. SEM of aeciospore formation in Puccinia bolleyana. Scan. Electron Microsc. 3:299-303.

Norton, R.A., L.L. Claypool, S.J. Leonard, P.A. Adrain, R.B. Fridley, and F.M. Charles. 1962. Mechanical harvest of sweet cherries. Calif. Agr. 16:8-10.

Patterson, S.E. 2001. Cutting loose. Abscission and dehiscence in Arabidopsis. Plant Physiol. 126:494-500.

Roberts, J.A., K.A. Elliott, and Z.H. Gonzalez-Carranza. 2002. Abscission, dehiscence and other cell separation processes. Annu. Rev. Plant Biol. 53:131-158.
Roberts, J.A., C.A. Whitelaw, Z.H. Gonzalez-Carranza, and M.T. McManus. 2000. Cell separation processes in plants-Models, mechanisms and manipulation. Ann. Bot. (Lond.) 86:223-235.

Ruzin, S.E. 1999. Plant microtechnique and microscopy. Oxford Univ. Press, New York, NY.

Sexton, R. and J.A. Roberts. 1982. Cell biology of abscission. Annu. Rev. Plant Physiol. 33:133-162.

Stösser, R., H.P. Rasmussen, and M.J. Bukovac. 1969. A histological study of abscission layer formation in cherry fruit during maturation. J. Amer. Soc. Hort. Sci. 94:239-243.

Takeda, F., G. Krewer, E.L. Andrews, B. Mullinix, and D.L. Peterson. 2008. Assessment of the V45 blueberry harvester on rabbiteye blueberry and southern highbush blueberry pruned to V-shaped canopy. HortTechnology 18:130-138.

U.S. Department of Agriculture. 1995. United States standards for grades of blueberries. U.S. Dept. Agr., Agr. Mktg. Serv., Washington, DC.

U.S. Department of Agriculture. 2012. Non-citrus fruits and nuts 2011 summary. National U.S. Dept. Agr., Agr. Stat. Serv., Washington, DC.

Webster, B.D. 1968. Anatomical aspects of abscission. Plant Physiol. 43:1512-1544.

Wittenbach, V.A. and M.J. Bukovac. 1972. An anatomical and histochemical study of abscission in maturing sweet cherry fruit. J. Amer. Soc. Hort. Sci. 97:214-219.

Wittenbach, V.A. and M.J. Bukovac. 1974. Cherry fruit abscission: Evidence for time of initiation and involvement of ethylene. Plant Physiol. 54:494-498. 